\title{
LIDERAZGO PEDAGÓGICO: DE LA SUPERVISIÓN AL ACOMPAÑAMIENTO DOCENTE
}

\author{
María Verónica Leiva-Guerrero ${ }^{1}$ y Camila Vásquez ${ }^{2}$
}

\begin{abstract}
RESUMEN
En la actualidad, la política educativa chilena plantea la necesidad de que los directivos escolares realicen procesos de acompañamiento a profesores, desde una perspectiva de liderazgo pedagógico para el fortalecimiento de las prácticas docentes y el aprendizaje de los estudiantes. Por ello, esta investigación tiene por objetivo caracterizar el acompañamiento docente que realizan directivos de establecimientos públicos de educación media de Valparaíso, Chile. Para cumplir dicho objetivo, se desarrolló un estudio cualitativo, con aplicación de entrevistas semiestructuradas a seis directivos y seis profesores de tres centros escolares. La información recogida fue procesada a través de un análisis de contenido de carácter deductivo. Los principales hallazgos permitieron observar que existe una presencia fluctuante y discrepancia entre los docentes y directivos en cuanto a los modelos de acompañamiento docente: mientras los directivos declaran aplicar modelos colaborativos e intervencionistas, los docentes señalan la aplicación mayoritaria de estos últimos, caracterizados por la entrega de directrices y aspectos resolutivos unidireccionales. La presencia fluctuante de categorías de acompañamiento docente de los tres modelos: intervencionista, colaborativo y facilitador, evidencia el proceso de transición que viven actualmente los establecimientos escolares, que transitan desde la supervisión al acompañamiento docente.
\end{abstract}

Conceptos clave: acompañamiento docente, directivos, liderazgo pedagógico, modelos de acompañamiento, supervisión.

\section{PEDAGOGICAL LEADERSHIP: FROM SUPERVISING TO SUPPORTING TEACHERS}

ABSTRACT

Currently, Chilean educational policy presents the need for school leaders to carry out processes to accompany teachers from a pedagogical leadership perspective, to strengthen teaching practices and student learning. For this reason, this research aims to characterize the teaching accompaniment carried out by directors of public secondary education schools in Valparaíso. To achieve this objective, a qualitative study was developed, with the application of semi-structured interviews to six directors and six teachers from three schools. The information collected was processed through a deductive content analysis. The main findings allowed to observe that there is a fluctuating presence and discrepancy between teachers and directors on the models of teacher accompaniment. While directors declare to apply both collaborative and interventionist models, teachers indicate that the latter is the most frequently applied, wherein the directors provide

1 Pontificia Universidad Católica de Valparaíso, Valparaíso, Chile. Contacto: veronica.leiva@pucv.cl

2 PontificiaUniversidad Católica de Valparaíso, Valparaíso, Chile. Contacto: camila.vasquez@pucv.cl 
guidelines and unidirectional resolutive aspects. This fluctuating presence of teaching support categories of the three models: interventionist, collaborative and facilitator, demonstrates the process of transition experienced by the schools, from supervision to teacher support.

Key concepts: accompaniment, accompaniment models, directors, pedagogical leadership, supervision, teacher.

\section{Introducción}

En los últimos años, las investigaciones han sostenido que, dentro de las múltiples variables internas que inciden en la mejora de la calidad educacional, el desempeño docente se posiciona como un factor de alto impacto. Este se asocia con el concepto de calidad e intrínsecamente hace referencia a aquellos profesores con buenos logros académicos y que obtienen resultados importantes en los aprendizajes de sus estudiantes (Villalobos, 2011), vale decir, mayor autonomía y autorregulación permanente en la instalación de significados profundos para adoptar un comportamiento estratégico ante la realización de actividades en diversos contextos (GonzálezGómez, et. al, 2018; Torres-Soto \& Vallejo-Ruiz, 2018). Por otra parte, Araneda (2018) plantea la necesidad de un sistema escolar de calidad "que aliente a niños, niñas y jóvenes a desarrollar sus capacidades y talentos, de manera que cada estudiante cuente con herramientas que les permitan construir sus proyectos de vida" (p. 8).

Sabemos que el desempeño docente dentro de aula no es el único factor que afecta la calidad de los aprendizajes: el liderazgo que ejerce el director en la escuela también tiene una influencia significativa en ellos (Day et al., 2010; Hallinger \& Wang, 2015). Por tanto, es deber de cada institución educativa articular y gestionar la labor docente y directiva para promover el mejoramiento continuo de las prácticas en el aula (Parra y Matus, 2016; Román, 2015).

En tal sentido, los líderes escolares no solo deben gestionar procesos administrativos, sino también otros referidos a la enseñanza y el aprendizaje, aspectos clave para la trasformación de la escuela, a fin de dar un salto desde la eficacia a la capacidad interna de mejora 
(Barber y Mourshed, 2008; Pont, Nusche \& Moorman, 2008). Lo anterior encuentra respaldo en diversos estudios que posicionan, específicamente, al liderazgo pedagógico como una variable de impacto, cuyo efecto se encontraría en torno al 25\% de la varianza total de las evaluaciones entre las escuelas (Anderson, 2010; Bolívar, 2010a; Hallinger y Heck, 2014; Horn y Marfán, 2010; Leiva, Montecinos y Aravena, 2016; López, 2010; Murillo y HernándezCastilla, 2015; Printy, 2010; Sarasola y Da Costa, 2016; Vaillant y Rodríguez, 2016).

Autores como Leithwood, Day, Sammons, Harris y Hopkins (2006) plantean que el liderazgo pedagógico implica que los establecimientos escolares, además de focalizar sus tareas en lograr altos niveles de aprendizaje en los estudiantes, requieren de:

- las prácticas orientadas al desarrollo del currículo,

- el desarrollo de todas las personas que conforman la comunidad educativa,

- la evaluación de los estudiantes y docentes,

- un trabajo con propósitos y metas compartidas entre la comunidad educativa,

- el análisis para la toma de decisiones sustentados en datos, y

- la generación de condiciones organizacionales, bajo un enfoque de colaboración (Arlestig \& Tornsen, 2014; Gajardo y Ulloa, 2016).

Además, prácticas relacionadas con el apoyo pedagógico a docentes son esenciales para un proceso de mejoramiento escolar efectivo y continuo (Johnston, Kaufman $\&$ Thompson, 2016; Louis, Leithwood, Wahlstrom \& Anderson, 2010).

Como complemento, la literatura especializada ha destacado el alto grado de influencia que evidencia el liderazgo pedagógico de los líderes escolares en el ejercicio docente, lo que repercute consecuentemente en las oportunidades de aprendizaje de todas y todos los estudiantes (Johnston et al., 2016; Kelemen, en prensa; Weinstein y Muñoz, 2012). Sin embargo, diversos estudios han corroborado que el ejercicio de un liderazgo centrado en lo pedagógico, se ve obstaculizado por la existencia de ciertas rutinas 
burocrático-administrativas, la continuidad y permanencia de modelos pedagógicos intervencionistas, la falta de tiempo, y de estrategias consensuadas y sistemáticas que orienten los procesos formativos de la institución escolar (Garbanzo y Orozco, 2010; Krichesky y Murillo, 2011; Martínez \& González, 2010; Rivera, 2010).

Al respecto, Anderson (2010) plantea que el líder educativo, es decir, el director, sigue evadiendo la corresponsabilidad que tiene su quehacer profesional en el aprendizaje del estudiantado, delegando esta tarea en otros miembros del equipo directivo, especialmente en los jefes de la Unidad Técnica Pedagógica (UTP) (Quiroga y Aravena, 2017). Esto lleva a que, en la actualidad, existan equipos directivos que adolecen del conocimiento pedagógico necesario para asumir un efectivo liderazgo pedagógico (Mellado, Chaucono y Villagra, 2017). A ello se suma la instalación, desde la política educativa chilena, del Marco para la buena dirección y liderazgo escolar (MBD) (2015) y de los Estándares indicativos de desempeño para los establecimientos educacionales y sus sostenedores (2014), que promueven el ejercicio de un liderazgo pedagógico. Estos instrumentos demandan la implementación de acciones de acompañamiento docente, que fomenten el desarrollo profesional de los profesores.

Este proceso debe caracterizarse por la generación de vínculos de confianza y comunicación efectiva, incentivos primarios para potenciar el mejoramiento de la práctica pedagógica y los procesos de aprendizaje de los estudiantes (Ministerio de Educación de Chile, Mineduc, 2014, 2015, 2018; Román y Murillo, 2008). Hargreaves $\&$ Fink (2007) señala que la confianza es un factor fundamental debido a que posibilita que las personas puedan trabajar de forma cooperativa, impactando positivamente en la manera en que se llevan a cabo los procesos de aprendizaje de los estudiantes en la escuela. Para esto se requiere de una estrecha interdependencia (Bryk $\&$ Schneider, 2002, citado en Tschannen-Moran, 2014; Peña, Weinstein $\&$ Raczynski, 2018) entre los actores involucrados y la disposición a depender de otro, confiando en que este cumplirá con obligaciones y expectativas importantes para la tarea compartida de educar a los estudiantes (Robbins, 2004). En este sentido, el acompañamiento de directivos debe proveer a los docentes de respeto interpersonal, 
respaldo pedagógico, retroalimentación, soporte técnico y una reflexión continua para la mejora de su desempeño, incorporando nuevas estrategias y procedimientos evaluativos para potenciar el aprendizaje de los estudiantes (Martínez y Jugo, 2014).

Asumir esta mirada pedagógica-colaborativa, que la actual política educativa nacional demanda a los líderes escolares, requiere de un escenario que promueva las responsabilidades y el aprendizaje mutuo entre docentes y directivos, donde exista un trabajo interpedagógico transformacional y crítico que permita cuestionar y actualizar las ideologías pedagógicas de enseñanza y aprendizaje (Anderson, 2010; Aziz, 2017; Bolívar, 2010a, 2010b; Elmore, 2010; Macbeath \& Nempster, 2009; Mellado et al., 2017).

En función de lo expuesto, esta investigación pretende identificar y describir los modelos de acompañamiento que implementan los directivos de centros escolares de educación media de la Región de Valparaíso a sus docentes.

\section{Acompañamiento docente}

El acompañamiento docente debe ser entendido como aquella acción que evoluciona de la supervisión educativa. En este sentido, Maureira (2015) lo plantea como una modalidad de trabajo que busca "caminar junto con los actores escolares en un diálogo horizontal y permanente, y en un contexto de confianza mutua con el fin de enfrentar, en conjunto, desafíos profesionales y educativos que son significativos" (p. 2). Además, corresponde a un proceso de mediación formativa, basado en una relación de ayuda, en la que se propicia el crecimiento profesional y emocional con el propósito de llegar a una meta o de conseguirla conjuntamente (Osto, 2006; Planela, 2009).

Esta herramienta de mediación formativa, según Martínez y González (2010), permite la cualificación de las prácticas, relaciones y procesos de gestión de aprendizajes desde la perspectiva comunitaria, vale decir, es un marco cohesionador e integrador de los procesos orientados a la profesionalización del perfil y práctica de los docentes para: 
asesorar personalmente al docente en su ámbito de trabajo, en su práctica cotidiana y a partir de sus necesidades específicas; es continuo y sostenido; es intencional, organizado y sistemático; se da por medio del diálogo, de la relación horizontal, de la interacción, la disposición personal y el compromiso (Montero, 2011, p. 72).

Sin embargo, para ello es necesario establecer una diferencia entre lo que es el acompañamiento docente y el enfoque del mismo. Al respecto, Cavalli (2006) distingue entre:

Acompañamiento docente: es la estrategia central que brinda soporte técnico y afectivo (emocional-ético y efectivo) para impulsar el proceso de cambio en las prácticas de los docentes. Está centrado en el desarrollo de las capacidades profesionales, a partir de la asistencia técnica, el diálogo y la promoción de la reflexión del maestro sobre su práctica pedagógica.

Enfoque del acompañamiento: corresponde al aprendizaje cooperativo entre pares, en donde se responde a una lógica de aprendizaje continuo. El facilitador que acompaña, es un maestro que ha tenido una trayectoria profesional exitosa. Entonces, el facilitador comparte con el docente su propia experiencia, a la vez que se enriquecen ambos. La relación facilitadora/docente está basada en la confianza mutua, la tolerancia y empatía (Cavalli, 2006, citado por Rodríguez-Molina, 2011, p. 262).

Al respecto, estudios señalan que los dispositivos de formación fundados en el aprendizaje autónomo, horizontal y colaborativo resultan más efectivos para lograr cambios en las prácticas de enseñanza (Ingvarson, Meiers \& Bebáis, 2005; Navarro \& Verdisco, 2000). Por tanto, el acompañamiento docente no consiste en eventos o instancias aisladas en una institución, mucho menos en una etapa con fases predeterminadas y acotadas. Se debe concebir como un proceso dinámico, sistemático, sostenido, holístico e interdisciplinario, que se debe asumir desde supuestos constructivistas y teoría sociocrítica, siendo la realidad del contexto el punto de partida y de convergencia de las acciones político-pedagógicas en la gestión de procesos de 
formación, de innovación y de cambio (Martínez y González, 2010; Segovia, 2010; Segovia, Bolívar-Botía, Luengo, Hernández y García, 2005).

\subsection{Modelos de acompañamiento docente}

Nieto (2001) propone tres modelos de acompañamiento docente centrados en elementos como las interacciones, el vínculo, la confianza, el trabajo en equipo, relaciones interpersonales, de poder y la declaración de las necesidades de apoyo dentro de las escuelas (Martínez y González, 2010). En la Tabla 1 se presentan dichos modelos, complementados con la visión de Segovia (2010) y Vezub y Alliaud (2012).

Tabla 1

Modelos educacionales de acompañamiento docente

\begin{tabular}{|c|c|c|c|}
\hline Dimensiones & $\begin{array}{l}\text { Modelo de } \\
\text { intervención }\end{array}$ & $\begin{array}{l}\text { Modelo de } \\
\text { facilitación }\end{array}$ & $\begin{array}{l}\text { Modelo de } \\
\text { colaboración }\end{array}$ \\
\hline $\begin{array}{l}\text { Concepción } \\
\text { epistemológica }\end{array}$ & Servicio técnico. & Apoyo. & Mutua formación. \\
\hline $\begin{array}{l}\text { Enfoque prioritario } \\
\text { de la intervención }\end{array}$ & $\begin{array}{l}\text { Ente resolutivo. } \\
\text { Punto de vista del } \\
\text { observador, asesor } \\
\text { y/o acompanante. }\end{array}$ & $\begin{array}{l}\text { Asesor orientador. } \\
\text { Docente debe } \\
\text { encontrar sus } \\
\text { propias soluciones. }\end{array}$ & $\begin{array}{l}\text { Figura de coach. } \\
\text { Construcción } \\
\text { con junta y } \\
\text { colaborativa, } \\
\text { interdependiente y } \\
\text { recíproca. }\end{array}$ \\
\hline $\begin{array}{l}\text { Finalidad de la } \\
\text { intervención }\end{array}$ & Remedial. & $\begin{array}{l}\text { Enriqueced ora- } \\
\text { preventiva. }\end{array}$ & Cooperadora. \\
\hline Relación & $\begin{array}{l}\text { Directiva, dominada } \\
\text { por el asesor } \\
\text { (diagnóstico y } \\
\text { prescripción } \\
\text { experta). }\end{array}$ & $\begin{array}{l}\text { De apoyo, dominada } \\
\text { por el docente } \\
\text { (asesor como } \\
\text { recurso para el } \\
\text { profesor). }\end{array}$ & $\begin{array}{l}\text { Colaborativa, } \\
\text { dominada por } \\
\text { ambos agentes } \\
\text { (toma de decisiones } \\
\text { en conjunto). }\end{array}$ \\
\hline
\end{tabular}

Fuente: Elaboración propia sobre la base de Nieto (2001), Segovia (2010) y Vezub \& Alliaud (2012).

\subsubsection{Modelo de intervención}

En este modelo se mantiene una significación vinculada con sistemas de supervisión y gestión de los niveles educativos, definiéndose un tipo de acompañamiento educativo centrado en el punto de vista del acompañante (Vezub y Alliaud, 2012). Este último es quien interpreta y define la realidad vivenciada por el docente acompañado, 
presentándose como un experto en el área pedagógica (Segovia, 2010). Desde esta posición, se establece una relación jerarquizada, que implica una conducta directiva orientada a la revisión, identificación y diagnóstico de los problemas del docente, para ofrecer una serie de remediales, es decir, prescribir lineamientos pedagógicos a seguir de forma unidireccional. Nieto (2001) declara que este tipo de acompañamiento se refleja en la metáfora del médico que diagnostica una dolencia y receta un remedio a su paciente.

\subsubsection{Modelo de facilitación}

Define un tipo de acompañamiento educativo centrado en el punto de vista del docente acompañado. En este modelo, el acompañante debe generar instancias que promuevan la reflexión del docente, para evitar entregar prescripciones. Se trabaja desde una perspectiva de servicio, donde el acompañante, mediante actividades conjuntas, se presenta como un recurso para el docente en la identificación, comprensión y formulación de problemáticas en el ejercicio de su profesión. Se induce al autodescubrimiento de soluciones y propuestas para mejorar sus prácticas pedagógicas (Segovia, 2010). Según Nieto (2001), este tipo de acompañamiento se asocia con la metáfora del psiquiatra, que escucha a su paciente y trata de guiarlo para que encuentre por sí mismo la solución a su problema.

\subsubsection{Modelo de colaboración}

Acompañamiento educativo basado en la interdependencia entre el acompañante y el docente acompañado. En esencia, se presenta una visión de formación mutua y recíproca, donde la toma de decisiones relativa a la resolución de problemas es consensual, ejercida por el facilitador y por el profesor en condiciones de igualdad de estatus y responsabilidad compartida, siendo la influencia bilateral y paritaria (Segovia, 2010; Vezub y Alliaud, 2012). Según Nieto (2001), este modelo de acompañamiento se refleja en la metáfora del colega o amigo crítico, alguien en quien se deposita confianza y con quien se puede trabajar en el marco de una relación sincera y mutuamente satisfactoria. 


\subsection{Equipos directivos y acompañamiento docente en Chile}

Desde el año 2014, el concepto de acompañamiento se ha posicionado con más fuerza dentro de las instituciones educativas de Chile. Esto se visualiza en los convenios de desempeño, elaborados desde el Mineduc, firmados por los directores participantes en los concursos de la Alta Dirección Pública y que asientan fuertemente el acompañamiento docente, bajo la perspectiva del desarrollo profesional.

En este sentido, Yana y Adco (2018) afirman que, si el objetivo es mejorar el desempeño docente, entonces se debe atender prioritariamente el acompañamiento a los docentes. Desde esta perspectiva, el Mineduc y el Centro de Perfeccionamiento, Experimentación e Investigaciones Pedagógicas (CPEIP) plantean este acompañamiento como "el proceso de inducción, acompañamiento y promoción de los docentes, coherentes con las necesidades personales, profesionales e institucionales, caracterizado por un clima de confianza y comunicación efectiva, incentivo para potenciar el mejoramiento de la práctica pedagógica" (Mineduc, 2018, p. 15).

Lo anterior adquiere relevancia, pues diversos estudios han corroborado que las estrategias como la asesoría al docente, la capacitación continua del profesorado y el apoyo de los directivos, son los cimientos de un efectivo proceso de enseñanza y aprendizaje (Mineduc, 2013; Román y Murillo, 2008; Villalobos, 2011). No obstante, el uso que se dé a este proceso al interior de los centros escolares dependerá exclusivamente de las concepciones que los líderes escolares tengan sobre el mismo.

En concordancia con lo anterior, la variedad de investigaciones acerca del acompañamiento docente determinan este proceso como una estrategia cohesionadora e integradora, la cual permite un intercambio tanto de experiencias como de conocimientos, orientados al perfeccionamiento de la práctica educativa, y donde el acompañante — por medio de la observación, valoración y retroalimentación del trabajo del profesorado—, enseña, aconseja, guía y ayuda al docente acompañado sobre la base de un conjunto de estrategias, procedimientos, reflexión conjunta y colaborativa (Aziz, 
2017; Román, 2015; Terrero, Legañoa y García, 2018; Yana y Adco, 2018).

Por último, es posible señalar que lo que busca el acompañamiento docente, desde las acciones y estrategias de los líderes escolares, es la formación de docentes con pensamiento crítico, promotores de acciones para comprender y cuestionar la realidad y ayudar a transformarla para el bien común.

\section{Método}

La investigación se abordó desde un diseño cualitativo fenomenológico de casos múltiples (Cebreiro y Fernández, 2004; Feagin, Orum \& Sjoberg, 1991; Pérez, 1994; Stake, 2005). Los datos se obtuvieron en el contexto de un proyecto de investigación más amplio sobre acompañamiento docente.

Considerando el tipo de estudio se plantean las siguientes interrogantes:

- ¿Qué modelos de acompañamiento docente están aplicando los directivos en los centros escolares de educación pública?

- ¿Existen diferencias entre directivos y profesores respecto de los tipos de modelos de acompañamiento docente aplicados en los centros escolares?

\subsection{Participantes}

Participaron del estudio 12 profesionales, seis profesores de educación media y seis directivos, en igual proporción, de tres centros escolares públicos de la ciudad de Valparaíso. Los directivos tienen los cargos de directores y jefes de UTP. Para no hegemonizar los discursos, se hizo la selección de directivos (director y jefe de UTP) y docentes de Lenguaje y Matemáticas, de segundo año de enseñanza media de establecimientos municipales.

La selección de participación fue por criterio de conveniencia (Flick, 2015) y, por voluntad de los directivos y profesores para ser entrevistados. Antes de proceder a la realización de las entrevistas los 
participantes firmaron un consentimiento informado. En la Tabla 2 se entrega el detalle de la caracterización de los participantes.

Tabla 2

Caracterización de participantes

\begin{tabular}{clcc}
\hline Participante & \multicolumn{1}{c}{ Especialidad } & $\begin{array}{c}\text { Años } \\
\text { docencia }\end{array}$ & $\begin{array}{c}\text { Años cargo } \\
\text { en el centro }\end{array}$ \\
\hline D1 & Profesor de educación técnico profesional. & 23 & 4 \\
\hline UTP1 & Profesor de Biología y Ciencias Naturales. & 22 & 18 \\
\hline PL1 & Licencia en Educación Lengua Castellana & 6 & - \\
\hline PM1 & Ingeniero en Biotecnología. & 2 & - \\
\hline D2 & Profesor de Química y Ciencias. & 22 & 23 \\
\hline UTP2 & Profesor de Estado en Educación & 10 & 4 \\
& Matemática. & 3 & - \\
\hline PL2 & Profesor de Lenguaje y Comunicación. & 7 & - \\
\hline PM2 & Profesor de Matemática. & 11 & 3 \\
\hline D3 & Profesor de Educación Básica. & 33 & 3 \\
\hline UTP3 & Profesor de Matemática. & 16 & - \\
\hline PL3 & Profesor de Lenguaje y Comunicación. & 28 & - \\
\hline PM3 & Profesora de Matemática. & & \\
\hline
\end{tabular}

Fuente: Elaboración propia.

\subsection{Procedimientos e instrumentos}

Los datos utilizados se obtuvieron a través de la aplicación de entrevistas semiestructuradas (Flick, 2004) a directores, jefes de UTP y docentes. Las entrevistas se realizaron en los tres centros escolares y fueron grabadas en audio y posteriormente transcritas para su análisis. Cada entrevista tuvo una duración aproximada entre 29 y 54 minutos (Tabla 3). 
Tabla 3

Aplicación de entrevistas

\begin{tabular}{cll}
\hline Participante & \multicolumn{1}{c}{ Lugar de entrevista } & \multicolumn{1}{c}{ Duración entrevista } \\
\hline D1 & Oficina director & 45 minutos y 56 segundos \\
\hline UTP1 & Oficina UTP & 29 minutos y 4 segundos \\
\hline PL1 & Sala de entrevistas & 45 minutos y 27 segundos \\
\hline PM1 & Salón de clases & 47 minutos y 31 segundos \\
\hline D2 & Oficina director & 31 minutos y 32 segundos \\
\hline UTP2 & Oficina UTP & 54 minutos y 28 segundos \\
\hline PL2 & Biblioteca & 49 minutos y 54 segundos \\
\hline PM2 & Biblioteca & 29 minutos y 5 segundos \\
\hline D3 & Oficina director & 1 hora y 13 minutos \\
\hline UTP3 & Oficina UTP & 31 minutos y 41 segundos \\
\hline PL3 & Sala de profesores & 26 minutos y 42 segundos \\
\hline PM3 & Salón de clases & 41 minutos y 24 segundos
\end{tabular}

Fuente: Elaboración propia.

\subsection{Análisis}

Las transcripciones de las entrevistas fueron analizadas con apoyo del software NVivo en su versión número 11. Se aplicó análisis de contenido de carácter deductivo (Mayring, 2000), respecto de los modelos de acompañamiento docente de la adaptación realizada de Nieto (2001), Segovia (2010) y Vezub y Alliaud (2012).

Las categorías a priori establecidas de acuerdo con los autores, permitieron la asignación de segmentos específicos a cada una de ellas, determinando así — desde una mirada más cuantitativa de análisis-, la presencia latente de modelos de acompañamiento docente de cada establecimiento. En la Tabla 4 se describen las categorías deductivas para el análisis de los modelos de acompañamiento pedagógico. 
Tabla 4

Categorías para análisis de los modelos de acompañamiento docente

\begin{tabular}{lll}
\hline \multicolumn{1}{c}{ Dimensión } & \multicolumn{1}{c}{ Tipo de modelo } & \multicolumn{1}{c}{ Categorías } \\
\hline Modelos de & Intervencionista & Iniciativas desde directivos. \\
acompañamiento & & Enfoque de intervención resolutiva. \\
docente. & Finalidad remedial. \\
& Relación directiva. \\
\cline { 2 - 3 } & Facilitador & Iniciativa desde profesores. \\
& Enfoque de intervención reflexiva. \\
& Finalidad preventiva. \\
& Relación de apoyo. \\
\cline { 2 - 3 } & Iniciativas consensuadas. \\
& Enfoque de intervención conjunta. \\
& Finalidad coayuda. \\
& Relación colaborativa. \\
\hline
\end{tabular}

Fuente: Elaboración propia.

Para validar las codificaciones de las categorías de modelos de acompañamiento docente señaladas en la Tabla 4, dos investigadores cruzaron sus codificaciones, estableciendo consenso en aquellas discrepancias. Posteriormente, un tercer investigador auditó las codificaciones asignadas.

\section{Resultados}

En relación con los modelos de acompañamiento se codificaron 194 segmentos para los directivos y 107 para los profesores. Los directivos señalan un tránsito de modelos de acompañamiento docente de un modelo intervencionista a uno colaborativo, a partir de generar espacios para la reflexión entre pares, producto del proceso de acompañamiento docente instalado en la escuela, lo que concordaría con un modelo colaborativo $(N=75)$ :

darles los tiempos también de reflexión entre los pares... ehh... hemos estado trabajando este año el tema de que sean acompañados entre pares... entre los mismos docentes, ya sea del mismo departamento o, también, de otro departamento (D2).

A su vez, se observa un rol jerárquico de experto-médico, que revisa y diagnostica problemas del docente para prescribir soluciones pedagógicas, muy propio de un modelo de acompañamiento docente intervencionista $(N=74)$ : 
yo le digo por ejemplo "¿sabe qué? su planificación está muy... ehh... elevada en comparación a lo que planificó el departamento" ... cuando yo conozco a mi estudiante, y sé que no podemos pasar a un nivel ehh... de... análisis... ahí yo le pongo un... un... no un paralé pero le digo, un alto al profesor (UTP2).

En cambio, los profesores expresan la presencia mayoritaria de un modelo de acompañamiento intervencionista $(N=55)$ :

me pregunta... ¿en qué va profesora? —mira, sabes que con esto del paro ellos, me atrasé, voy a saltarme esto, voy a mover esto otro-, no, no, no, yo creo que lo mejor que puedes hacer es tomar este contenido, y verlo de esta forma (PM3), por sobre modelos facilitadores $(N=25)$ y colaborativos $(N=27)$.

Específicamente, en el modelo intervencionista, la categoría "Enfoque de intervención resolutiva", donde prevalece el punto de vista y la interpretación del asesor-acompañante, presenta más segmentos codificados ( $N=34$, directivos y $N=33$, profesores), como se observa en la siguiente textualidad:

lo primero, bueno obviamente la UTP hizo como un análisis del contenido que uno explica, que la técnica, que el explicarlo, que eso estaba bien, mal y todo eso, y... después ya pasamos como, por ejemplo, digamos a... a lo más doméstico dentro de la sala de clases... que el alumno esté pendiente, que el alumno esté trabajando, que no esté escuchando música, que esté... observando a la pizarra (PM2).

Finalmente, desde el discurso de los actores escolares entrevistados, se evidencian tres categorías coincidentes que presentan escasas codificaciones. Del modelo intervencionista, la subdimensión "Finalidad remedial" $(N=2)$, que refiere al objetivo de las conversaciones entre docentes y directivos, que apuntan a la entrega de prescripciones al trabajo pedagógico de manera vertical; del modelo facilitador, la subcategoría "Iniciativa desde profesores" $(N=3)$, relacionada con el levantamiento de necesidades desde los docentes, como una manera de atender a requerimientos personales, 
pedagógicos y/o institucionales; y del modelo colaborador; y la subdimensión "Relación colaborativa" $(N=2)$, respecto de los vínculos de confianza, reciprocidad, empatía y corresponsabilidad, establecidos entre directivos y docentes, como se observa en las Tablas 5 y 6 .

Tabla 5

Modelos de acompañamiento docente implementados, declarados por directivos.

\begin{tabular}{|c|c|c|c|c|c|c|c|c|c|c|c|c|c|}
\hline \multirow[b]{2}{*}{ 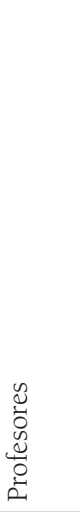 } & \multicolumn{4}{|c|}{$\begin{array}{c}\text { Modelo } \\
\text { intervencionista }\end{array}$} & \multicolumn{4}{|c|}{ Modelo facilitador } & \multicolumn{4}{|c|}{ Modelo colaborativo } & \\
\hline & 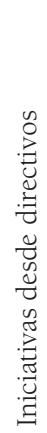 & 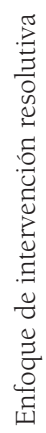 & 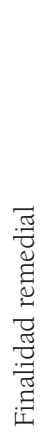 & 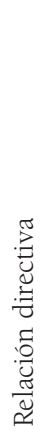 & 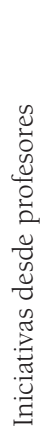 & 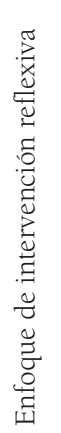 & 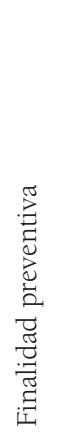 & 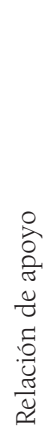 & 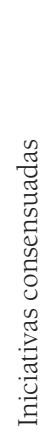 & 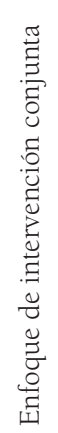 & 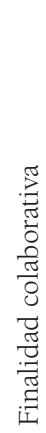 & 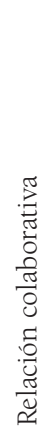 & \\
\hline D1 & 3 & 5 & - & 6 & - & 1 & - & 6 & 8 & 4 & 2 & 2 & \\
\hline UTP1 & 6 & 2 & - & - & 1 & - & 1 & 1 & 7 & 1 & 1 & - & \\
\hline D2 & - & 2 & - & 4 & 1 & 2 & 2 & 4 & 1 & 6 & 2 & - & \\
\hline UTP2 & 1 & 19 & 2 & 1 & 1 & 5 & - & 6 & 2 & 11 & - & - & \\
\hline D3 & 2 & 4 & - & 8 & - & 3 & 3 & - & 6 & 3 & - & - & \\
\hline UTP3 & 6 & 2 & - & 1 & - & 3 & 5 & - & 7 & - & 3 & - & \\
\hline \multirow[t]{2}{*}{ Total } & 18 & 34 & 2 & 20 & 3 & 14 & 11 & 17 & 4 & 25 & 8 & 2 & $\begin{array}{r}19 \\
4\end{array}$ \\
\hline & & 74 & & & & & 45 & & & & 75 & & \\
\hline
\end{tabular}

Fuente: Elaboración propia.

Podemos analizar, además de los resultados de la Tabla 5, que en los equipos directivos, los directores señalan mayor presencia de un modelo de acompañamiento docente colaborativo $(N=43)$ que los jefes de UTP $(N=32)$. Mientras que los jefes de UTP declaran en sus discursos una aplicación mayor del modelo intervencionistas $(N=39)$, que los directores $(N=34)$. 
Tabla 6

Modelos de acompañamiento docente implementados, declarados por profesores

\begin{tabular}{|c|c|c|c|c|c|c|c|c|c|c|c|c|}
\hline \multirow[b]{2}{*}{$\begin{array}{l}\mathscr{O} \\
\stackrel{0}{0} \\
\stackrel{\mathscr{W}}{0} \\
\stackrel{0}{0}\end{array}$} & \multicolumn{4}{|c|}{$\begin{array}{c}\text { Modelo } \\
\text { intervencionista }\end{array}$} & \multicolumn{4}{|c|}{ Modelo facilitador } & \multicolumn{4}{|c|}{ Modelo colaborativo } \\
\hline & 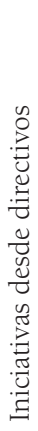 & 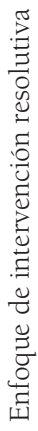 & 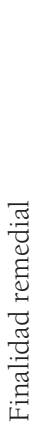 & 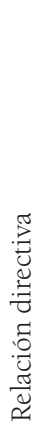 & 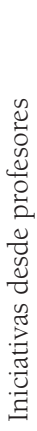 & 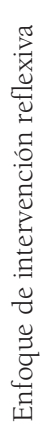 & 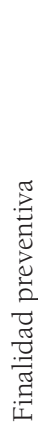 & 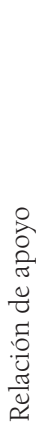 & 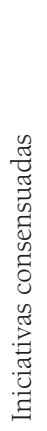 & 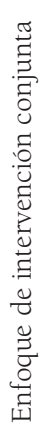 & 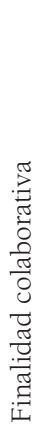 & 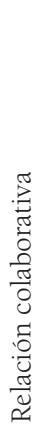 \\
\hline PLl & 3 & 5 & 1 & 3 & - & 2 & - & - & 3 & - & - & - \\
\hline PM1 & 1 & 7 & - & 4 & - & 4 & 3 & - & 7 & 1 & - & - \\
\hline PL2 & 2 & 3 & 1 & 1 & 2 & - & 3 & - & 5 & 1 & - & - \\
\hline PM2 & - & 8 & - & 4 & - & - & 1 & - & 3 & 1 & - & - \\
\hline PL3 & - & 4 & 1 & - & - & 2 & - & 2 & 2 & - & 1 & - \\
\hline PM3 & - & 6 & - & 1 & 3 & 2 & - & 1 & 1 & 1 & 1 & - \\
\hline \multirow{2}{*}{ Total } & 6 & 33 & 3 & 13 & 5 & 10 & 7 & 3 & 21 & 4 & 2 & - \\
\hline & \multicolumn{4}{|c|}{55} & \multicolumn{4}{|c|}{25} & \multicolumn{4}{|c|}{27} \\
\hline
\end{tabular}

Fuente: Elaboración propia.

Por otra parte, podemos visualizar en las Tablas 5 y 6 que el proceso de acompañamiento docente es una estrategia consensuada entre directivos y docentes ( $N=40$, directivos; $N=21$ docentes), en términos de horarios de visita al aula:

Se realiza una planificación semestral del acompañamiento docente, donde se define en conjunto día y hora de la visita al aula (D2),

pero con baja participación de los profesores en los procesos de definición y sentido del acompañamiento docente (categoría "Iniciativas desde los docentes": $N=3$ en directivos y $N=5$ en docentes), señalando:

No se ha dado... como te explico... a ver... una instancia de conversación, donde se nos pida nuestra opinión, de cómo 
podemos implementar el acompañamiento, de cuáles son nuestras necesidades, de qué nos gustaría... (PM1).

Igualmente, existe una muy baja relación colaborativa $(N=2$ en directivos y $N=0$ en docentes), lo que afirmaría que los procesos de acompañamiento docente desde los equipos directivos pueden tener una intencionalidad participativa y colaborativa, pero en la práctica no la ejercen.

\section{Discusión y conclusiones}

Se evidencia desde el discurso emanado por directivos y docentes la presencia de categorías para los tres modelos de acompañamiento docente: intervencionista, colaborativo y facilitador. Sin embargo, hay una mayor predominancia del modelo intervencionista, en el cual los directivos otorgan a sus docentes directrices y aspectos resolutivos unidireccionales. Las relaciones dominantes son en base a una jerarquía entregada por los cargos de liderazgo, con escasa confianza. Al respecto, Maureira (2015) plantea que más que acompañar a los docentes, se busca el control y la supervisión de lo que hacen, generando desconfianza en el profesorado y su resistencia al proceso de acompañamiento docente.

Lo anterior se contrapone con los lineamientos de un liderazgo pedagógico efectivo y eficaz, caracterizado en la literatura internacional, como también en las políticas públicas del Mineduc para la mejora de los sistemas escolares y el avance hacia una educación de calidad (Leiva et al., 2016). En esta la confianza que se genere entre los distintos actores de la escuela debe ser relevante, ya que posibilita que las personas puedan trabajar de forma cooperativa y participativa, impactando positivamente en la manera en que se llevan a cabo los procesos de enseñanza y aprendizaje (Hargreaves $\&$ Fink, 2007).

En este plano, los líderes educativos asumen un papel fundamental, sobre todo en los centros escolares más vulnerables, donde estudiantes y profesores pasan a ser el motor de la gestión directiva (Tapia et al., 2011, citado en Razeto, 2017). Es decir, cuando 
las relaciones interpersonales están alimentadas por la confianza, los vínculos entre profesores y directivos se constituyen en parte de los cimientos de la organización escolar que posibilita el trabajo colectivo (Bryk \& Schneider, 2002) y facilitan la mejora del funcionamiento para desarrollar un proceso sostenible de cambio asentado en el vínculo social.

Las categorías de acompañamiento que se declaran en segundo lugar, corresponden al modelo colaborativo, específicamente en lo que tiene que ver con las decisiones conjuntas entre directivos y docentes respecto de la planificación y la ejecución de procesos, como visitas al aula, etapas del proceso y utilización de herramientas de evaluación de la observación de clases. Bryk y Schneider (2002, en Razeto, 2017) observan al respecto, consecuencias clave para la organización escolar, como la creación de una cultura de colaboración que realce el compromiso entre todos los agentes educativos con el centro educativo, en una búsqueda constante de innovación en todos los ámbitos que influyen en la gestión del mismo. Además, se facilita la estructura de trabajo para la toma de decisiones en el conjunto de la comunidad de profesionales, con apoyo para la enseñanza del profesor, lo cual tiene directa influencia en el ámbito instruccional y aprendizaje de los estudiantes.

Siguiendo con las categorías ligadas al modelo colaborativo, se ha intentado establecer un enfoque de intervención conjunta, toda vez que se han intencionado estrategias como el acompañamiento entre pares y redistribuciones de tareas pedagógicas, a través de equipos especializados en ciertas temáticas ligadas al desarrollo curricular y evaluativo institucional. Al respecto, múltiples estudios aseguran que los modelos de acompañamiento con un enfoque de colaboración, entre los líderes escolares y los equipos de docentes, marcan las potencialidades y la calidad de los resultados alcanzados por los estudiantes y el impacto futuro en el desarrollo sostenible de los entornos educativos (Barber y Mourshed, 2008; Bolívar, López y Murillo, 2013; Horn y Marfán, 2010; Leithwood, 2009; Leithwood \& Louis, 2011; Medina \& Gómez, 2014). De aquí la importancia de generar una cultura de comunidad profesional en la escuela, desde la reflexión crítica conjunta y la socialización de prácticas pedagógicas 
entre los docentes y directivos escolares. Para ello, ambos, deben estar involucrados en el análisis y comprensión de lo que ocurre en el aula (Mineduc, 2019).

Las categorías del modelo facilitador que se mencionan mayormente son: "Relación de apoyo", en donde el acompañante se presenta como un facilitador de procesos y/o de recursos al acompañado; "Enfoque de intervención reflexiva", caracterizado por instancias de reflexión que son guiadas por el acompañante; y "Finalidad preventiva", toda vez que la meta del trabajo reflexivo es obtener una panorámica de los posibles obstáculos pedagógicos por encontrar, para plantear soluciones viables y diversas entre ambas partes.

Esta presencia fluctuante de categorías de acompañamiento docente de los tres modelos: intervencionista, colaborativo y facilitador, evidencia según Vezub (2011) el proceso de transición que viven los establecimientos: desde la supervisión al acompañamiento docente.

Por otra parte, se evidencia discrepancia en las declaraciones de ambos grupos entrevistados. En la declaración de los directivos, se observa una presencia predominante de los modelos colaborativo e intervencionista, en cambio los profesores expresan la presencia de un modelo intervencionista por sobre uno facilitador o colaborador.

Esta conjugación híbrida de modelos, según Segovia (2010), se debe a que las acciones que permiten visualizar la implementación de los mismos pueden variar desde acciones puntuales y/o poco pertinentes — que no llegan a desarrollar e instalar capacidades en la comunidad escolar-, hasta sistemas de apoyo democráticos, colaborativos, éticos y contextualizados, que permitan, a través de acciones y propuestas técnicas adecuadas, una transformación auténtica, desde adentro.

A modo de sugerencias, incorporar modelos de acompañamiento facilitadores y colaborativos en los centros educativos, crearía las condiciones profesionales necesarias para el 
trabajo colaborativo y el desarrollo de una comunidad de aprendizaje al interior de la escuela, por medio de la reflexión crítica, propositiva colectiva y permanente (Hatch, Hill \& Roegman, 2016).

Además, se desprende la necesidad de capacitar a los equipos directivos para instalar en los centros educativos públicos modelos reales de acompañamiento docente, que propendan al trabajo metacognitivo, entendido como la capacidad de conocer, analizar y reflexionar acerca de los mecanismos, prácticas y procesos personales de aprendizaje profesional docente (Rottemberg y Anijovich, 2007). Sin embargo, este trabajo de capacitación debe ser realizado de forma contextualizada, entregando los correspondientes apoyos, formación, estructuras de soporte, elementos catalizadores y mediadores para la mejora (Corcoran, Fuhrman \& Belcher, 2001; Johnston et al., 2016; Mourshed, Chijioke y Barber, 2011;), pues si el foco de las nuevas políticas educativas está en el fortalecimiento de la calidad de la educación pública en Chile, se requiere de directivos capaces de desarrollar competencias holísticas innovadoras para los sistemas educativos (Aziz, 2017). No obstante, sin un cambio real en la cultura de los centros escolares respecto del aporte significativo que tiene el acompañamiento docente para el desarrollo profesional y la creación de comunidades de aprendizaje, desde un liderazgo con foco en lo pedagógico (gestión del currículo y de los procesos de enseñanza y aprendizaje), se seguirán implementando modelos intervencionistas en las escuelas, con una lógica desde la supervisión, centrada en la evaluación, más que modelos de acompañamientos centrados en la colaboración que nutran la profesión docente.

\section{Referencias}

Anderson, S. (2010). Liderazgo directivo: claves para una mejor escuela. Psicoperspectivas, 9(2), 34-52. https://doi.org/10.5027/ psicoperspectivas-Vol9-Issue2-fulltext-127

Araneda, P. (2018). Tarea de todos, hacia una visión compartida de la calidad de la educación. Santiago de Chile: Agencia de Calidad de la Educación.

Arlestig, H. \& Tornsen, M. (2014). Classroom observations and supervisionessential dimensions of pedagogical leadership. International Journal of Educational Management, 28(7), 856-868. https://doi.org/10.1108/ ijem-01-2014-0001 
Aziz, C. (2017). Acompañamiento pedagógico desde el nivel intermedio: aportes para la formación (Informe técnico $\mathrm{N}^{\circ} 4$ ). Santiago de Chile: Centro de Liderazgo para la Mejora Escolar. Recuperado de https://www. lidereseducativos.cl/wp-content/uploads/2017/11/IT_CA_L6.pdf

Barber, M. \& Mourshed, M. (2008). Cómo hicieron los sistemas educativos con mejor desempeño del mundo para alcanzar sus objetivos. Recuperado de www.oei.es/historico/pdfs/documento_preal41.pdf

Bolívar, A. (2010a). El liderazgo educativo y su papel en la mejora: una revisión actual de sus posibilidades y limitaciones. Psicoperspectivas, 9(2), 9-33. https://doi.org/10.5027/psicoperspectivas-Vol9-Issue2fulltext-112

Bolívar, A. (2010b). ¿Cómo un liderazgo pedagógico y distribuido mejora los logros académicos? Revisión de la investigación y propuesta. Magis, Revista Internacional de Investigación en Educación, 3(5), 79-106. Recuperado de https://revistas.javeriana.edu.co/index.php/MAGIS/ article/view/3528

Bolívar, A., López, J., y Murillo, F.J. (2013). Liderazgo en las instituciones educativas. Una revisión de líneas de investigación. Revista Fuentes, 14, 15-60. Recuperado de http://institucional.us.es/revistas/fuente/14/ Firma\%20invitada.pdf

Bryk, A. S., \& Schneider, B. L. (2002). Trust in schools: A core resource for improvement. The Rose series in sociology. New York, NY: Russell Sage Foundation.

Cebreiro, B. y Fernández, M. (2004). Estudio de casos. En F. Salvador Mata, J. L. Rodríguez Diéguez y A. Bolívar-Botia (Eds.), Diccionario enciclopédico de didáctica (pp. 1-764), Málaga: Aljibe.

Cavalli, M. (2006). La evaluación de la práctica pedagógica. Revista Iberoamérica de Educación, 35(4), 29-46. Recuperado de rieoei.org/ deloslectores/877Cavalli.PDF

Corcoran, T. B., Fuhrman, S. H., \& Belcher, C. L. (2001). The district role in instructional improvement. Recuperado de http://repository.upenn.edu/ gse_pubs/6.

Day, C., Sammons, P., Leithwood, K., Hopkins, D., Harris, A., Gu, Q., \& Brown, E. (2010). Ten strong claims about successful school leadership. Nottingham: NCSL.

Educación de Calidad para Todos: un asunto de Derechos Humanos (2007). Documento de discusión sobre políticas educativas en el marco de la II Reunión Intergubernamental del Proyecto Regional de Educación para América latina y el Caribe. Buenos Aires: EPT/PRELAC. 
Elmore, R. (2010). Mejorando la escuela desde la sala de clases. Santiago de Chile: Fundación Chile.

Feagin, J., Orum, A., \& Sjoberg, G. (Eds.). (1991). A case for case study. Chapel Hil, London: University of North Carolina Press.

Flick, U. (2004). Introducción a la investigación cualitativa. Madrid: Morata.

Flick, U. (2015). El diseño de investigación cualitativa. Madrid: Morata.

Gajardo, J. y Ulloa J. (2016). Liderazgo pedagógico, conceptos y tensiones (Nota Técnica $N^{\circ} 6$ ). Concepción: Centro de Liderazgo para la Mejora Escolar. Recuperado de https://www.lidereseducativos.cl/wp-content/ uploads/2017/01/NT-6.pdf

Garbanzo, G. y Orozco, V. (2010). Liderazgo para una gestión moderna de procesos educativos. Revista Educación, 32(1), 15-29. https://doi. org/10.15517/revedu.v34i1.495

González-Gómez, C., Veas, A., Fernández, F., Jover, I., Navarro., Priore, A., ..., y García, D. (2018). Los procesos cognitivos de autorregulación clave para la mejora de la calidad en los diferentes aprendizajes en el ámbito universitario: la atención ejecutiva. Una experiencia en el aula. En R. Roig-Vila (Coord.), J. Antolí, A. Lledó y N. Pellín (Eds.), Memorias del Programa de Redes-I3CE. Convocatoria 2017-18 (pp. 847-864). Alicante: Instituto de Ciencias de la Educación (ICE), Universidad de Alicante.

Hallinger, P. y Heck, R. (2014). Liderazgo colaborativo y mejora escolar: comprendiendo el impacto sobre la capacidad de la escuela y el aprendizaje de los estudiantes. REICE. Revista Iberoamericana sobre Calidad, Eficacia y Cambio en Educación, 12(4), 71-88. Recuperado de http://www.redalyc.org/articulo.oa?id=55131688004

Hallinger, P. \& Wang, W. C. (2015). Assessing instructional leadership with the principal instructional management rating scale. Dordrecht: Springer.

Hargreaves, A. y Fink, D. (2007). Principios de un liderazgo sostenible. Revista Padres y Maestros, 310, 17-21. Recuperado de https://revistas.comillas. edu/index.php/padresymaestros/article/view/1659/2597

Hatch, T., Hill, K., \& Roegman, R. (2016). Investigating the role of instructional rounds in the development of social networks and district-wide improvement. American Educational Research Journal, 53(4), 1022-1053. https://doi.org/10.3102/0002831216653205

Horn, A. y Marfán, J. (2010). Relación entre liderazgo educativo y desempeño escolar: revisión de la investigación en Chile. Psicoperspectivas, 9(2), 82-104. https://doi.org/10.5027/psicoperspectivas-Vol9-Issue2fulltext-116 
Ingvarson, L., Meiers. M., \& Bebáis, A. (2005). Factors affecting the impact of professional development programs on teachers' knowledge, practice, student outcomes \& efficacy. Education Policy Analysis Archives, 13(10), 1-20. https://doi.org/10.14507/epaa.v13n10.2005

Johnston, W. R., Kaufman J. H., \& Thompson, L. E. (2016). Support for instructional leadership. Recuperado de http://www.wallacefoundation. org/knowledgecenter/Documents/Support-for-InstructionalLeadership.pdf

Kelemen, M. (en prensa). Focusing principal supervision on instruction. Lessons from three districts (Report). New: York: New Leaders publication.

Krichesky, G. y Murillo, J. (2011). Las comunidades profesionales de aprendizaje. Revista Iberoamérica sobre Calidad, Eficacia y Cambio en Educación, 9(1), 66-91. Recuperado de http://www.rinace.net/reice/ numeros/arts/vol9numl/art4.pdf

Leithwood, K., Day, C., Sammons, P., Harris, A., \& Hopkins, D. (2006). Successful school leadership. What it is and how it influences pupil learning. London: DfES.

Leithwood, K. (2009). ¿Cómo liderar nuestras escuelas? Aportes desde la investigación. Santiago de Chile: Fundación Chile.

Leithwood, K. \& Louis, K.S. (2011). Linking leadership to student learning: Empirical insights. San Francisco: Jossey Bass.

Leiva, M. V., Montecinos, C., y Aravena, F. (2016). Liderazgo pedagógico en directores nóveles en Chile: prácticas de observación de clases y retroalimentación a profesores. RELIEVE. Revista Electrónica de Investigación y Evaluación Educativa, 22(2), 1-17. http://dx.doi. org/10.7203/relieve.22.2.9459

López, P. (2010). El componente liderazgo en la validación de un modelo de gestión escolar hacia la calidad. Educação e Pesquisa, 36(3), 779-794.

Louis, K. S., Leithwood, K., Wahlstrom, K. L., \& Anderson, S. E. (2010). Learning from leadership: Investigating the links to improved student learning. Minnesota: The Wallace Foundation.

Macbeath, J. \& Nempster, N. (Eds.) (2009). Connecting leadership and learning. Principles for practice. London: Routledge.

Martínez, H. y González, S. (2010). Acompañamiento pedagógico y profesionalización docente: sentido y perspectiva. Ciencia y Sociedad, 35(3), 521-541. 
Martínez, N. y Jugo, M. (2014). Protocolo de acompañamiento pedagógico. Recuperado de http://www2.minedu.gob.pe/digesutp/ formaciondeformadores/wpdescargas/2014/01_Protocolos_ acompamiento.pdf

Maureira, F. (2015). Acompañamiento ¿A escuelas o a las aulas? Cuadernos de Educación, 66, 1-10. Recuperado de http://mailing.uahurtado.cl/ cuaderno_educacion_66/pdf/articulo_3.pdf

Mayring, P. (2000). Qualitative content analysis. Forum Qualitative Social Research, 1(2), 1-10. http://dx.doi.org/10.17169/fqs-1.2.1089

Medina Rivilla, A. y Gómez Díaz, R. (2014). El liderazgo pedagógico: competencias necesarias para desarrollar un programa de mejora en un centro de educación secundaria. Perspectiva Educacional, 53(1), 91-113.

Mellado, M., Chaucono, J., y Villagra, C. (2017). Creencias de directivos escolares: implicancias en el liderazgo pedagógico. Psicologia Escolar e Educacional (São Paulo), 21(3), 541-548. http://dx.doi. org/10.1590/2175-3539/2017/0213111102.

Ministerio de Educación de Chile, Mineduc (2013). Programa directores para Chile. Santiago de Chile: Autor.

Ministerio de Educación de Chile, Mineduc (2014). Estándares indicativos de desempeño para los establecimientos educacionales y sus sostenedores. Santiago de Chile: Autor.

Ministerio de Educación de Chile, Mineduc (2015). Marco para la buena dirección y el liderazgo escolar. Santiago de Chile: Editora e Imprenta Maval.

Ministerio de Educación de Chile, Mineduc (2018). Orientaciones postulación y suscripción convenios de desempeño colectivo. Recuperado de http:// www.gestionyliderazgoeducativo.cl/gestioncalidad/asignacion/home/ img/Orienta2018.pdf

Ministerio de Educación de Chile, Mineduc (2019). Trabajo colaborativo y desarrollo profesional docente. Recuperado de https://bibliotecadigital. mineduc.cl/handle/20.500.12365/2266

Montero, C. (2011). Estudio sobre acompañamiento pedagógico. Experiencias, orientaciones y temas pendientes. En Consejo Nacional de Educación (Ed.), Hacia una propuesta de criterios de buen desempeño docente. Estudios que aportan a la reflexión, al diálogo y a la construcción concertada de una política educativa (pp. 71-347). Lima: CNE, FSM.

Mourshed, M., Chijioke, Ch., y Barber, M. (2011). Cómo continúan mejorando los sistemas educativos de mayor progreso en el mundo (PREAL Serie Documentos $N^{o}$ 61). Santiago de Chile. Recuperado de https:// 
es.slideshare.net/IlsePatio/cmo-continan-mejorando-los-sistemaseducativos-de-mayor-progreso-en-el-mundo

Murillo, F. J. y Hernández-Castilla, R. (2015). Liderazgo para el aprendizaje: ¿qué tareas de los directores y directoras escolares son las que más inciden en el aprendizaje de los estudiantes? RELIEVE. Revista Electrónica de Investigación y Evaluación Educativa, 21(1), 1-20. http:// dx.doi.org/10.7203/relieve.21.1.5015

Navarro, J. \& Verdisco, A. (2000). Teacher training in Latin America: Innovations and trends. (Technical Paper Series, $n^{\circ} 114$ ). Washington: InterAmerican Development Bank, Sustainable Development Department, Education Unit.

Nieto, J. (2001). Modelos de asesoramiento a organizaciones educativas. En J. D. Segovia (Coord.), Asesoramiento al centro educativo. Colaboración y cambio en la institución. (pp. 147-166). Barcelona: Octaedro-EUB.

NVivo en su versión número (11), (2019). Recuperado de http://www. qsrinternational.com/nvivo-spanish

Osto, E. (2006). Proyecto formativo. México, D.F: Ediciones S.T.J.

Planela, J. (2009). Ser educador. Entre la pedagogía y el nomadismo. Barcelona: Editorial Universitat Oberta de Catalunya.

Parra, V. y Matus, G. (2018). Usos de datos y mejora escolar: una aproximación a los sentidos y prácticas educativas subyacentes a los procesos de toma de decisiones. Calidad en la Educación, 45, 207-250. https://doi. org/10.31619/caledu.n45.25

Peña, J. Weinstein, J., y Raczynski, D. (2018). Construcción y pérdida de la confianza de docentes: Un análisis de incidentes críticos. Psicoperspectivas, 17(1), 1-13. https://doi.org/10.5027/ psicoperspectivas-vol17-issuel-fulltext-1174

Pérez, G. (1994). Investigación cualitativa. Retos, interrogantes y métodos. Madrid: La Muralla.

Pont, B., Nusche, D., \& Moorman, H. (2008). Improving school leadership. Volume 1: Policy and Practice. Recuperado de http://www.oecd.org/ dataoecd/32/12/44374889.pdf

Printy, S. (2010). Principals' influence on instructional quality: Insights from US schools. School Leadership and Management, 30(2), 111-126. https:// doi.org/10.1080/13632431003688005

Quiroga, M. y Aravena, F. (2017). Jefes de UTP noveles en Chile: desafíos del liderazgo pedagógico. Perspectiva Educacional, Formación de Profesores, 56(3), 76-97. https://doi.org/10.4151/07189729-Vol.56-Iss.3-Art.506 
Razeto, A. (2017). Más confianza para una mejor escuela: el valor de las relaciones interpersonales entre profesores y director. Cuadernos de Investigación Educativa, 8(1), 61-76. https://doi.org/10.18861/ cied.2017.8.1.2639

Rivera, L. (2010). Elementos conceptuales para el análisis de las prácticas de gestión educativa. En M. Guerra (Coord.), Gestión de la educación básica. Referentes, reflexiones y experiencias de investigación (pp. 71-86). México, D.F.: SEPUPN.

Robbins, S. (2004). Comportamiento organizacional. México, D.F.: Editorial Pearson.

Rodríguez-Molina, G. (2011). Funciones y rasgos del liderazgo pedagógico en los centros de enseñanza. Educación y Educadores, 14(2). Recuperado de https://educacionyeducadores.unisabana.edu.co/index.php/eye/ article/view/1921/2510

Román, M. (octubre, 2015). Liderazgo y eficacia escolar. Trabajo presentado en el $5^{\circ}$ Encuentro de Rectores, Bogotá, Colombia.

Román, M. y Murillo, F. J. (2008). La evaluación del desempeño docente: objeto de disputa y fuente de oportunidades en el campo educativo. Revista Iberoamericana de Evaluación Educativa, 1(2), 1-6. https://doi. org/10.15366/riee

Rottemberg, R. y Anijovich, R. (2007). Los docentes como diseñadores de la enseñanza. En M. Kap (Ed.), Estrategias de enseñanza y diseño de unidades de aprendizaje. Carpeta de trabajo (pp. 45-71). Buenos Aires: Universidad Virtual de Quilmes.

Sarasola, M. y Da Costa, C. (2016). Evaluando el liderazgo educativo centrado en los aprendizajes del alumnado. Educación, 25(49), 121-139. http:// dx.doi.org/10.18800/educacion.201602.008

Segovia, J., Bolívar-Botía, A., Luengo, F., Hernández, V. M., y García, R. (2005). Nuevas formas de asesorar y apoyar a los centros educativos. Revista Electrónica Iberoamericana Sobre Calidad, Eficacia y Cambio en Educación, 3(1), 382-402. Recuperado de http://www.redalyc.org/ articulo.oa?id $=55130138$

Segovia, J. (2010). Comprender y redireccionar las prácticas de asesoría. Revista Iberoamericana de Educación, 54, 65-83. https://doi. org/10.35362/rie540542

Stake, R. E. (2005). Investigación con estudio de casos. Madrid: Morata.

Tschannen-Moran, M. (2014). Trust matters: Leadership for successful schools (2nd ed). San Francisco, CA: Jossey-Bass. 
Tapia, C., Becerra, S., Mansilla, J., y Saavedra, J. (2011). Liderazgo de los directivos docentes en contextos vulnerables. Educación y Educadores, 14(2), 389-409. Recuperado de http://www.scielo.org.co/pdf/eded/ v14n2/v14n2a09.pdf

Terrero, M., Legañoa, M., y García, J. (2018). Modelo de gestión del acompañamiento pedagógico para maestro de primaria. Transformación, 14(1), 44-57. Recuperado de http://scielo.sld.cu/scielo.php?script=sci_ arttext\&pid=S207729552018000100005\&lng=es\&tlng=es

Torres Soto, A. y Vallejo Ruiz, M. (2018). Organización y funcionamiento de los agrupamientos en Educación Superior: concepciones de los estudiantes. Tendencias PedagóGicas, 32, 15-30. http://dx.doi. org/10.15366/tp2018.32.002

Vaillant, D. y Rodríguez, E. (2016). Prácticas de liderazgo para el aprendizaje en América Latina: un análisis a partir de PISA 2012. Ensaio: Avaliação e Políticas Públicas em Educação, 24(91), 253-274. http://dx.doi. org/10.1590/S0104-40362016000200001

Vezub, L. (2011). Las políticas de acompañamiento pedagógico como estrategia de desarrollo profesional docente. El caso de los programas de mentoría a docentes principiantes. Revista del IICE, 30, 109-132. Recuperado de http://revistascientificas.filo.uba.ar/index.php/iice/ article/view/149/111

Vezub, L. y Alliaud, A. (2012). Informe final: el acompañamiento pedagógico como estrategia de apoyo y desarrollo profesional de los docentes noveles. Recuperado de www.noveles.edu.uy/acompanamiento_pedagogico. pdf

Villalobos, X. (2011). Reflexión en torno a la gestión de aula y a la mejora en los procesos de enseñanza y aprendizajes. Revista Iberoamericana de Educación, 3(55), 1-7. https://doi.org/10.35362/rie5531590

Weinstein, J. y Muñoz, G. (2012). ¿Qué sabemos sobre directores de escuelas en Chile? Santiago de Chile: Fundación Chile y Pontificia Universidad Católica de Chile.

Yana, M. y Adco, H. (2018). Acompañamiento pedagógico y el rol docente en jornada escolar completa: caso instituciones educativas Santa Rosa y Salesianos de San Juan Bosco-Puno Perú. Revista de Investigaciones Altoandinas, 20(1), 137-148. http://dx.doi.org/10.18271/ria.2018.337

Recibido: 01/04/2019

Aceptado: 07/11/2019 